Review

\title{
Microbiome Associations of Therapeutic Enteral Nutrition
}

\section{Rajesh Shah ${ }^{1}$ and Richard Kellermayer ${ }^{2, *}$}

1 Department of Internal Medicine, Section of Gastroenterology, Baylor College of Medicine, Houston, TX, 77030, USA; E-Mail: rajeshs@bcm.edu

2 Department of Pediatrics, Section of Gastroenterology, Baylor College of Medicine and Texas Children’s Hospital, Houston, TX, 77030, USA

* Author to whom correspondence should be addressed; E-Mail: kellerma@bcm.edu; Tel.: +1-832-822-1051; Fax: +1-832-825-3633.

Received: 21 August 2014; in revised form: 8 October 2014 / Accepted: 21 October 2014 / Published: 21 November 2014

\begin{abstract}
One of the most effective forms of therapeutic enteral nutrition is designated as “exclusive enteral nutrition” (EEN). EEN constitutes the monotonous enteral delivery of complete liquid nutrition and has been most explored in the treatment Crohn's disease (CD), a form of inflammatory bowel disease. While EEN's mechanisms of action are not clearly understood, it has been shown to modify the composition of the intestinal microbiome, an important component of CD pathogenesis. The current literature on the intestinal microbiome in healthy individuals and CD patients is reviewed with respect to EEN therapy. Further investigations in this field are needed to better understand the role and potential for EEN in chronic human disorders.
\end{abstract}

Keywords: enteral nutrition; Crohn’s disease; microbiota; IBD; rheumatoid arthritis

\section{Introduction}

Therapeutic enteral nutrition describes the provision of defined nutritional support with the goal of treating a disease state. Despite a long history using therapeutic enteral nutrition, the critical mechanisms of action by which dietary changes can exert beneficial effects in specific human disorders are incompletely understood. Multiple hypotheses have been put forward in this regard, which include reduction in antigen exposure, overall nutritional repletion, improvement in intestinal barrier function, 
provision of micronutrients and reduction in dietary fat or carbohydrate, leading to reduced byproducts producing inflammation [1,2].

Exclusive enteral nutrition (EEN) is a subtype of therapeutic nutrition where dietary intake is confined to the persistent delivery of the same complete food preparation. Most commonly, EEN is achieved by liquid enteral formulas. Liquid formulations of EEN are typically divided into either being elemental or polymeric. Elemental formulas deliver a protein source as individual amino acids, and polymeric formulas provide intact protein. EEN has been most commonly employed to treat Crohn's disease (CD) [3]. CD is an incurable subtype of inflammatory bowel diseases (IBDs) characterized by transmural inflammation of the intestine anywhere from the mouth to the anus. The pathogenesis of CD is believed to involve the interaction between a defective intestinal barrier, environmental risk factors, the intestinal microbiome and host genetics [4,5]. Many of these biological systems, apart from genetics, are suspected to be modulated by EEN [2], which may explain its efficacy in treating CD. More recently, the microbiome related effects of this therapeutic nutrition are coming into focus. EEN has also been explored in the treatment of other autoimmune diseases, such as juvenile idiopathic arthritis [6] and ulcerative colitis [7], but these studies are rather limited. Therefore, this review will focus on CD with respect to the microbiome-related influences of EEN.

\section{Treatment of CD with EEN}

Induction and maintenance of remission (symptom free disease) are the current treatment goals for CD. The available medical therapies to achieve these goals include 5-aminosalicyclic acid (5-ASA) derivatives, immunosuppressive or immunomodulator agents (azathioprine, methotrexate), biologic medications (molecularly-engineered antibodies against key components of the human intestinal inflammatory cascade: infliximab, adalimumab, certolizumab, natalizumab, vedolizumab, etc.) or steroids (prednisone, budesonide). For severe CD, induction is typically achievable with either biologics or steroids. Thereafter, the continuation or transition to immunomodulators or biologic agents can substantiate remission. This approach, however, has limitations, including increased risk of adverse events (pancreatitis, hepatitis, neutropenia/bone marrow suppression) and potential long-term complications, such as a higher risk of malignancies [8,9]. Secondary to the limitations of conventional medical treatments, EEN has been explored as an adjunct or monotherapy for CD.

Since steroids are commonly used to induce remission, studies compared the efficacy of EEN to steroids during early CD treatment. Small, randomized trials found that children treated with elemental nutrition or steroids had similar rates of clinical improvement. Elemental EEN, however, was superior to steroids in terms of improved growth velocity $[10,11]$. Though these results were encouraging, the treatment durations were only 4-6 weeks, leaving questions about the potential for EEN as a maintenance therapy. Another limitation of these studies was non-compliance, since $8 \%-11 \%$ of children were unable to tolerate the elemental diet due to bad taste.

To overcome the limitations of elemental EEN, some trials have used polymeric formulas to induce or maintain remission in CD patients. Early investigations comparing polymeric formulations to steroids suggested that steroids were superior for inducing remission [12,13]; however, more recent work demonstrated equal efficacy of the two treatments $[14,15]$. Comparison between these trials is difficult, since they used different formulations of polymeric nutrition and for varied durations. Small cohort 
studies have examined if patients maintained on EEN (elemental or polymeric) remain in remission, but the results suggested that approximately $40 \%-60 \%$ will relapse within a year [16-18]. However, a significant proportion of this relapse rate may be attributed to discontinuation of EEN, which occurred in $20 \%-50 \%$ of the patients. These studies indicate that elemental and polymeric nutrition have equal therapeutic efficacy in CD, so either may be considered. Despite encouraging efficacy results for polymeric nutrition, high relapse rates likely arising from patient non-compliance and deliberate discontinuation due to taste limit EEN as a maintenance therapy for CD.

The discussed studies predominantly used clinical scores (Crohn's Disease Activity Index, CDAI) to determine treatment efficiency. More recently, the demonstration of intestinal mucosal healing is becoming a favored treatment endpoint [19]. Mucosal healing has been correlated with reduced risk of disease relapse, for example [20]. Steroids do not effectively induce mucosal healing, but several studies have shown that EEN can [21-24]. Borrelli et al. demonstrated in a randomized controlled trial of 37 children with CD that EEN induced mucosal healing in a greater proportion of patients compared to corticosteroids $(p<0.001)$ at 10 weeks. A single small cohort trial observed that polymeric nutrition induced mucosal healing by magnetic resonance enterography imaging [25]. Magnetic resonance enterography (MRE) can noninvasively detect small bowel mucosal damage with a high sensitivity [26,27]. These studies suggest that EEN may be an efficacious therapy for healing mucosal injury related to $\mathrm{CD}$, but definitive conclusions are limited by patients frequently receiving concomitant IBD medications (6-mercaptopurine, mesalamine, etc.). Further well-designed studies will be needed to confirm these findings while controlling for other confounding factors.

Based on the above, EEN appears to be as efficacious and perhaps even superior to steroids, for inducing remission in CD. EEN has several other benefits compared to steroids, including improved growth [10,11] and quality of life [28], optimized bone metabolism [29] and induction of mucosal healing [21-23]. Another common complication of CD is intestinal stricturing, which can necessitate intestinal resections. The risk of recurrence and subsequent surgeries remains high after resections [30]. Interestingly, a recent prospective cohort study from Japan indicated that even partial (50\% caloric requirements) delivery of EEN can significantly reduce the endoscopic (mucosal) recurrence of the disease at the surgical site after a year of surgery [31]. The overall need for re-operation tended to be less as well ( $p=0.08)$ in the partially EEN-treated group.

Despite the encouraging findings above, EEN has to be further explored to clearly determine its therapeutic potential as an induction and/or maintenance therapy for CD. The questions of its utility as a mono- versus combination therapy and as exclusive versus partial nutrition remain to be answered, as well. Community-based data from Spain suggested that physicians are frequently (63\%) recommending EEN to CD patients [32], but several barriers (poor long-term compliance, lack of optimized teaching, variable patient acceptance) create practical limitations for exploring the full potential of this promising treatment modality. Additionally, the mechanism of action for EEN would be important to define for the optimization of this practically challenging treatment, which carries a desirable side effect spectrum, compared to other available medical therapies. In the next section, we will review our limited understanding of EEN biology. 


\section{EEN Mechanisms of Action}

Several hypotheses have been proposed to explain the efficacy of EEN for CD. The most likely mechanisms include direct anti-inflammatory effects, improvement of intestinal barrier function and modulation of the intestinal microbiome (Figure 1).

Figure 1. Schematic representation of the gut microbiota, the intestinal epithelium and immune system; and how exclusive enteral nutrition (EEN) may affect these systems. Crohn's disease is associated with dysbiosis of the gut microbiota, increased permeability of the intestinal epithelium and dysregulated immunity. Studies suggest that EEN can alter the composition of the gut microbiota (A), reduce intestinal permeability through modulation of tight junctions either directly (B) or through modulating the intestinal microbiome (A) and downregulate the production of inflammatory cytokines either directly $(\mathrm{C})$ or through modulating the intestinal microbiome (A).

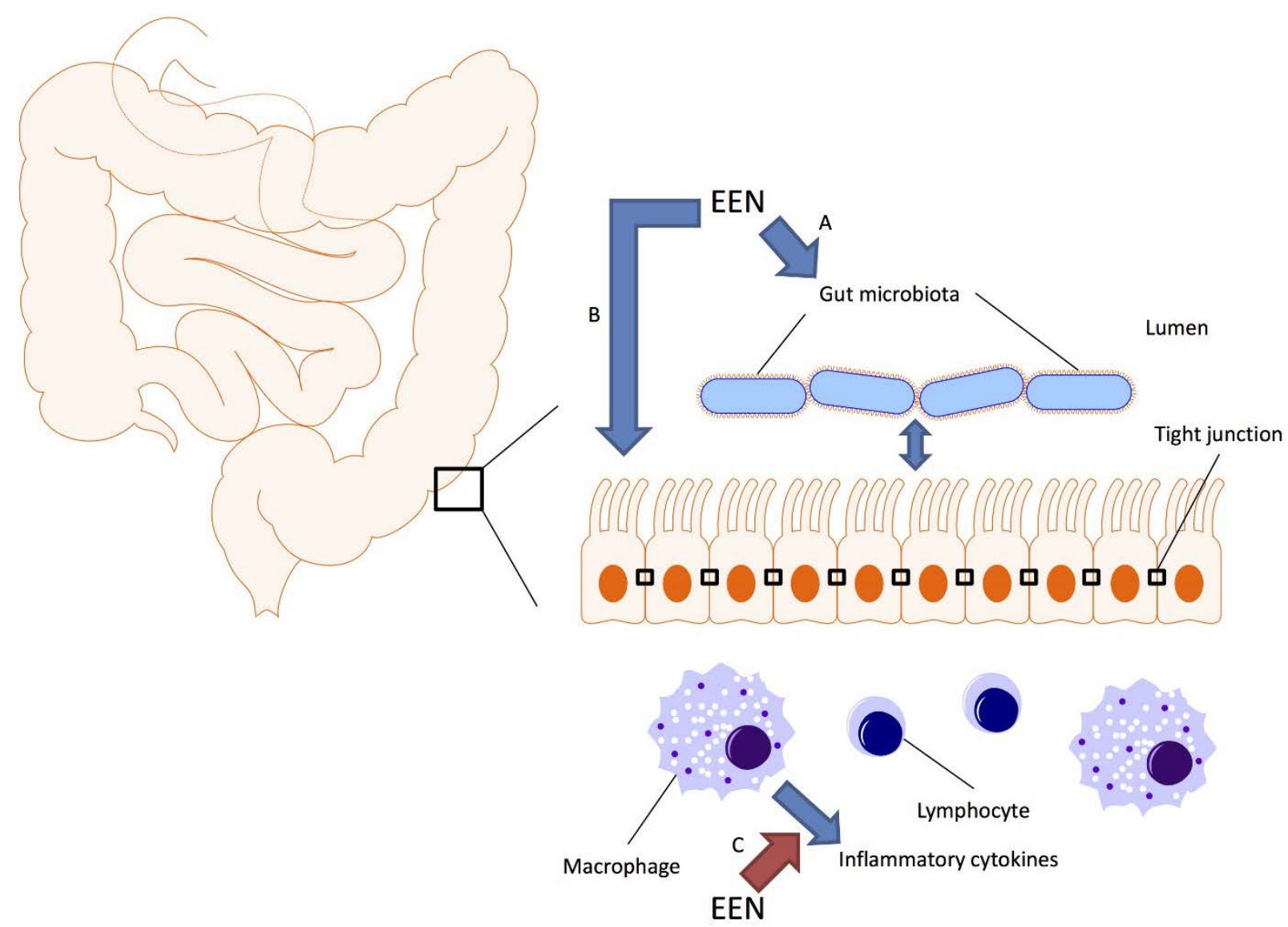

CD is characterized by increased production of multiple pro-inflammatory cytokines (TNF-alpha, IL-1, IL-6, etc.) [33]. Studies in children have shown that treatment with polymeric enteral nutrition can downregulate mucosal concentrations of these cytokines, as well as improve mucosal integrity [34]. Similar results were found using in vitro techniques, where pro-inflammatory signaling pathways were downregulated in the presence of polymeric formula [35,36]. These studies, however, were limited, since a precise molecular mechanism could not be defined, and the authors acknowledged the need for further work in this area. 
Mucosal barrier integrity is vital to the proper functioning of the intestines. Mucosal barrier function is maintained in part by structures designated as tight junctions, which join epithelial cells and participate in the regulation of ion, nutrient and water transport. In CD, the mucosal barrier is dysfunctional, which can be beneficially modified with EEN [37]. In vitro models using colonic epithelial cells have demonstrated that EEN can reduce intestinal permeability after the induction of colitis [38,39]. Interestingly, clinical studies have also shown that first-degree family members of CD patients have increased intestinal mucosal permeability when compared to non-IBD controls [40]. These findings suggest that patients with CD have intrinsic mucosal barrier defects, which could be modulated by EEN. However, the exact means by which EEN may improve mucosal permeability are not well understood.

Recent advances in sequencing technologies and bioinformatics have also allowed for examining the potential influence of EEN on the intestinal microbiome, which is another critically important biological system in the pathogenesis of $\mathrm{CD}$. We will next review the human gut microbiome and its potential importance in CD and EEN therapy.

\section{Microbiome Basics}

The human intestinal microbiome comprises a diverse, highly-interactive ecological system of bacteria, Archaea, viruses and fungi [41]. Classically, the intestinal microbiome was characterized by standard microbiological techniques, but those were inherently limited by the inability to culture over $60 \%$ of intestinal bacteria. Recently, investigators have leveraged the conserved 16S rRNA gene present in all bacteria to characterize the intestinal microbiome with sequencing techniques. This gene has taxon-specific variable regions, the sequencing of which can allow for bacterial classification. Additional techniques have been developed to sequence all genes present in a tissue sample (whole genome sequencing, WGS) and characterize the overall gene content attributed to microbes [42,43]. These techniques allow for bacterial classification and examination of overall gene content, but lack the ability to provide information about the actual biological activity or function of a specific microbiome. The functional activity of microbiomes is more specifically examined by the maturing fields of metatranscriptomics and metabolomics [42,44].

In parallel with the application of these new sequencing techniques, bioinformatic tools have been developed to process and interpret large datasets $[42,45,46]$. These tools enable the generation of common ecological measures (richness and diversity) to characterize the intestinal microbiome community. Richness describes the number of unique bacterial (or other microbial) species present in a sample, and diversity accounts for both the number and the relative abundance of the different species. As microbial clinical studies incorporate these metrics, investigators are finding significant associations with multiple diseases, such as obesity [47] and IBD [48].

Results from the American Human Microbiome Project (HMP) and European metagenomics of the human intestinal tract (MetaHIT) projects have provided composition and functional information for the healthy human microbiome [49,50]. These studies showed highly varying intestinal microbiome composition between subjects, but an overall similar functional capacity. From these and subsequent work, we gained an appreciation for the multitude of factors influencing microbiome composition (age, diet, sampling method). Microbiome composition appears to fluctuate rapidly early in life, remain relatively stable during adulthood and change again towards the seventh decade of life [41]. In addition 
to age, long-term dietary patterns strongly influence the intestinal microbiome [51-53]. Additionally, the intestinal microbiome can rapidly respond to short-term dietary interventions, as well, but reverts to its prior composition once the interventions cease [54-56]. Furthermore, the gut microbiome is different at the mucosal surface from that of luminal content. Therefore, the clinical sample type (feces versus mucosa) has to be taken into consideration [57,58], when planning and comparing microbiome studies of the gut specifically.

\section{EEN Effects on the Intestinal Microbiome}

A limited number of studies have described the intestinal microbiome of healthy subjects during EEN. Using a cross-over design, Whelan et al. examined the effect of fructooligosaccharide and fiber supplemented EEN on intestinal microbiota. They found that total intestinal bacterial counts were reduced during EEN [59]. However, this work utilized fluorescent in situ hybridization (FISH) techniques, which carry significant limitations compared to the current high-throughput technologies. Subsequently, the same group re-analyzed this data to describe the change in Faecalibacterium prausnitzii during EEN. F. prausnitzii is a commensal, Gram-positive bacterium with anti-inflammatory properties, which has been implicated in CD pathogenesis [60]. Their group found significant reductions in F. prausnitzii abundance during fiber supplementation, which demonstrated that diet can modulate specific bacterial concentrations [61]. Associations between F. prausnitzii, CD and EEN will be discussed in detail in the subsequent sections.

After characterizing the healthy human microbiome by state-of-the-art technology, investigators are now exploring the differences present in diseases. The term dysbiosis refers to the abnormal composition of the intestinal microbiome and has been studied in multiple disease states (obesity, diabetes, etc.). Reduced fecal microbiome richness [47] and diversity [62] were found in obese individuals. WGS revealed compositional differences between those with type 2 diabetes and non-diabetic controls, which may influence overall insulin resistance [63,64]. These and many other association studies indicate that abnormal composition and subsequent pathologic functioning of the microbiome may contribute to human disease.

\section{The Gut Microbiome of Crohn's Disease}

The intestinal microbiome of CD patients has been found to have overall dysbiosis compared to the datasets from HMP and MetaHIT [65,66]. Hansen et al. studied colon biopsy specimens from treatment-naive pediatric CD patients and detected significant reductions in diversity compared to healthy controls and patients with ulcerative colitis (UC, the other form of IBD) [65]. Gevers and colleagues examined both stool and mucosal samples from a very large cohort of treatment-naive pediatric CD patients and found modest overall differences compared to controls, which was increased in patients with higher disease activity (Table 1) [66]. Detailed bioinformatic analyses revealed further functional differences between CD and controls. CD microbiomes expressed pathways involved in inflammation and had reduced functionality related to amino acid, carbohydrate and nucleotide metabolism [66]. Other investigations in treatment-experienced CD patients have identified similar functional changes $[67,68]$. 
Table 1. Description of mucosal microbiota changes found in treatment-naive Crohn's disease $[65,66]$. Several bacterial taxa are increased or decreased compared to non-IBD controls. * Changes in Faecalibacterium were inconsistent across studies, with one study reporting an increase and the other a decrease.

\begin{tabular}{cc}
\hline \multicolumn{2}{c}{ Crohn's Disease } \\
\hline Increased & Decreased \\
\hline Enterobacteriaceae & Bacteroidales \\
Pasteurellaceae & Clostridiales \\
Fusobacteriaceae & Erysipelotrichaceae \\
Neisseriaceae & Bifidobacteriaceae \\
Veillonellaceae & Coriobacteriaceae \\
Gemellaceae & Faecalibacterium \\
Faecalibacterium & \\
\hline
\end{tabular}

The role of specific bacteria in CD is less understood. The difficulties in making a definite conclusion about single species are well demonstrated by the example of $F$. prausnitzii. Previous observations noted reduced concentrations of $F$. prausnitzii in the terminal ileum of CD patients and higher concentrations correlated with reduced risk of recurrence [69,70]. These studies, however, were confounded by examining treatment-experienced patients, where microbiome composition may have been modified by the treatments themselves [67,71]. Explorations in treatment-naive patients led to conflicting results on F prausnitzii, with one small cohort reporting higher abundance of the bacterium compared to controls [65], as opposed to a lower abundance found in a recent large-scale study [66].

These studies emphasize the complexity of the microbiome in IBD and underscore the need for careful planning (i.e., accounting for confounding factors, recruiting adequate number of patients) to enable the detection of meaningful differences. These limitations have to be taken into account when interpreting the available literature on EEN-microbiome effects in CD patients.

\section{EEN Induced Microbiome Changes in CD Patients}

Only a few studies have examined the microbial effects of EEN in CD patients. Lionetti et al. reported results from a small case series of nine pediatric CD patients treated with polymeric enteral nutrition. They noted that eight of nine children obtained clinical remission, and all experienced significant shifts in intestinal microbiome composition [72]. These shifts were determined using the gel electrophoresis banding pattern, resulting in a limited depth of interrogation when compared to high-throughput technologies. Similar results were found in a study of six children with CD treated with EEN, as well as correlations between specific bacterial taxa and the degree of inflammation [73]. In a recently published work on EEN treatment for pediatric CD (utilizing gel electrophoresis and quantitative real-time PCR), investigators found decreases in overall microbiome diversity and reductions in F. prausnitzii abundance during EEN therapy [74]. These findings challenge the previous notions of increased microbiome diversity associated with health and higher concentrations of $F$. prausnitzii associated with reduced CD activity. In accordance with the difficulties for interpreting the role of $F$. prausnitzii in CD, some observations indicate that regardless of disease activity, the abundance of it remains low in CD [75] and can even further decrease upon clinical improvement with elemental enteral therapy [76]. Clearly, further 
work is required to understand the role of F. prausnitzii in CD and IBD in general. Such controversies emphasize the challenges of microbiome research, even in the current era of advanced technology.

To gain a better understanding of EEN's effects on the intestinal microbiome, our lab explored this interaction in a mouse model. We set up the hypothesis that it is the monotonous nature of EEN that is critical to its therapeutic effect and not the liquid nature or low antigenicity of it. Healthy mice were assigned to either a single chow or alternating chows. This was meant to mimic either EEN or a more free (liberalized) diet, respectively. After 20 days of the monotonous or the alternating diet feeding, mice were given dextran sulfate sodium (DSS), an intestinal irritant that induces acute large bowel inflammation (colitis) and is an accepted model of human IBD. Mice given the single chow diets, similar to EEN, had higher overall microbiome diversity compared to alternating chow fed mice [77]. These results were consistent with the prior human findings described by Leach et al. [73]. However, the results of Gerasimidis and colleagues [74] oppose the observations in the murine experiment. Regardless, the mice provided alternating chow were also more susceptible to intestinal injury from DSS, supporting an association between reduced microbiome diversity and susceptibility to colitis in mammals. This observation is also consistent with the reduction of microbiome diversity in IBD patients [65,66]. Altogether, our murine model work supported the hypothesis that the monotonous nature of EEN may be a key component to its efficacy. Epidemiologic observations correlating agricultural import with the incidence of CD in a European country further substantiated the possible validity of the "monotonous diet” hypothesis [77]. Further high-throughput studies will need to explore the effects of EEN on microbiome composition in both healthy people and CD patients to elucidate the exact effects of this nutritional therapy in health and disease.

\section{Future Considerations and Conclusions}

Several randomized clinical trials have shown the efficacy for partial and exclusive elemental or polymeric nutritional therapies to induce clinical improvement and even remission in CD. EEN appears to be superior to steroids with respect to the induction of mucosal healing during induction therapy and may have the potential to provide long-term remission in some cases of CD. The highlighted limitations of the existing clinical studies and the practical challenges for this drastic nutritional therapy warrant further intense work towards the optimization of this treatment modality. Though the mechanism(s) of action of nutritional therapy remains unknown, the detected changes in the intestinal microbiome composition and function induced by EEN provide new routes for research. The findings from such investigations may facilitate the development of novel treatment strategies, not only for CD, but for other autoimmune disorders, as well.

\section{Acknowledgments}

The authors would like to thank the Gutsy Kids Fund, including philanthropic donation from the Karen and Brock Wagner family and other generous families. 


\section{Author Contributions}

Rajesh Shah: conception, drafting and editing of the manuscript; Richard Kellermayer: conception, drafting, editing and final approval of the manuscript.

\section{Conflicts of Interest}

The authors declare no conflict of interest.

\section{References}

1. Critch, J.; Day, A.S.; Otley, A.; King-Moore, C.; Teitelbaum, J.E.; Shashidhar, H.; Committee, N.I. Use of enteral nutrition for the control of intestinal inflammation in pediatric Crohn disease. J. Pediatr. Gastroenterol. Nutr. 2012, 54, 298-305.

2. Nahidi, L.; Day, A.S.; Lemberg, D.A.; Leach, S.T. Paediatric inflammatory bowel disease: A mechanistic approach to investigate exclusive enteral nutrition treatment. Scientifica 2014, 2014, doi:10.1155/2014/423817.

3. Zachos, M.; Tondeur, M.; Griffiths, A.M. Enteral nutritional therapy for induction of remission in Crohn's disease. Cochrane Database Syst. Rev. 2007, 1, doi:10.1002/14651858.CD000542.

4. Xavier, R.J.; Podolsky, D.K. Unravelling the pathogenesis of inflammatory bowel disease. Nature 2007, 448, 427-434.

5. Kellermayer, R. Epigenetics and the developmental origins of inflammatory bowel diseases. Can. J. Gastroenterol. 2012, 26, 909-915.

6. Berntson, L. Anti-inflammatory effect by exclusive enteral nutrition (EEN) in a patient with juvenile idiopathic arthritis (JIA): Brief report. Clin. Rheumatol. 2014, 33, 1173-1175.

7. González-Huix, F.; Fernández-Bañares, F.; Esteve-Comas, M.; Abad-Lacruz, A.; Cabré, E.; Acero, D.; Figa, M.; Guilera, M.; Humbert, P.; de León, R.; et al. Enteral versus parenteral nutrition as adjunct therapy in acute ulcerative colitis. Am. J. Gastroenterol. 1993, 88, 227-232.

8. Siegel, C.A.; Marden, S.M.; Persing, S.M.; Larson, R.J.; Sands, B.E. Risk of lymphoma associated with combination anti-tumor necrosis factor and immunomodulator therapy for the treatment of Crohn's disease: A meta-analysis. Clin. Gastroenterol. Hepatol Off. Clin. Pract. J. Am. Gastroenterol. Assoc. 2009, 7, 874-881.

9. Long, M.D.; Martin, C.F.; Pipkin, C.A.; Herfarth, H.H.; Sandler, R.S.; Kappelman, M.D. Risk of melanoma and nonmelanoma skin cancer among patients with inflammatory bowel disease. Gastroenterology 2012, 143, 390-399.

10. Sanderson, I.R.; Udeen, S.; Davies, P.S.; Savage, M.O.; Walker-Smith, J.A. Remission induced by an elemental diet in small bowel Crohn's disease. Arch. Dis. Childhood 1987, 62, 123-127.

11. Thomas, A.G.; Taylor, F.; Miller, V. Dietary intake and nutritional treatment in childhood Crohn's disease. J. Pediatr. Gastroenterol. Nutr. 1993, 17, 75-81.

12. Malchow, H.; Steinhardt, H.J.; Lorenz-Meyer, H.; Strohm, W.D.; Rasmussen, S.; Sommer, H.; Jarnum, S.; Brandes, J.W.; Leonhardt, H.; Ewe, K.; et al. Feasibility and effectiveness of a defined-formula diet regimen in treating active Crohn's disease. European cooperative Crohn's disease study III. Scand. J. Gastroenterol. 1990, 25, 235-244. 
13. Lochs, H.; Steinhardt, H.J.; Klaus-Wentz, B.; Zeitz, M.; Vogelsang, H.; Sommer, H.; Fleig, W.E.; Bauer, P.; Schirrmeister, J.; Malchow, H. Comparison of enteral nutrition and drug treatment in active Crohn's disease. Results of the european cooperative Crohn's disease study IV. Gastroenterology 1991, 101, 881-888.

14. Gonzalez-Huix, F.; de Leon, R.; Fernandez-Banares, F.; Esteve, M.; Cabre, E.; Acero, D.; Abad-Lacruz, A.; Figa, M.; Guilera, M.; Planas, R.; et al. Polymeric enteral diets as primary treatment of active Crohn's disease: A prospective steroid controlled trial. Gut 1993, 34, 778-782.

15. Ruuska, T.; Savilahti, E.; Maki, M.; Ormala, T.; Visakorpi, J.K. Exclusive whole protein enteral diet versus prednisolone in the treatment of acute Crohn's disease in children. J. Pediatr. Gastroenterol. Nutr. 1994, 19, 175-180.

16. Takagi, S.; Utsunomiya, K.; Kuriyama, S.; Yokoyama, H.; Takahashi, S.; Iwabuchi, M.; Takahashi, H.; Takahashi, S.; Kinouchi, Y.; Hiwatashi, N.; et al. Effectiveness of an 'half elemental diet' as maintenance therapy for Crohn's disease: A randomized-controlled trial. Aliment. Pharmacol. Ther. 2006, 24, 1333-1340.

17. Duncan, H.; Buchanan, E.; Cardigan, T.; Garrick, V.; Curtis, L.; McGrogan, P.; Barclay, A.; Russell, R.K. A retrospective study showing maintenance treatment options for paediatric Crohn's disease in the first year following diagnosis after induction of remission with EEN: Supplemental enteral nutrition is better than nothing! BMC Gastroenterol. 2014, 14, doi:10.1186/1471-230X-14-50.

18. Knight, C.; El-Matary, W.; Spray, C.; Sandhu, B.K. Long-term outcome of nutritional therapy in paediatric Crohn's disease. Clin. Nutr. 2005, 24, 775-779.

19. Peyrin-Biroulet, L.; Bressenot, A.; Kampman, W. Histologic remission: The ultimate therapeutic goal in ulcerative colitis? Clin. Gastroenterol. Hepatol. Off. Clin. Pract. J. Am. Gastroenterol. Assoc. 2014, 12, 929-934.

20. Ardizzone, S.; Cassinotti, A.; Duca, P.; Mazzali, C.; Penati, C.; Manes, G.; Marmo, R.; Massari, A.; Molteni, P.; Maconi, G.; et al. Mucosal healing predicts late outcomes after the first course of corticosteroids for newly diagnosed ulcerative colitis. Clin. Gastroenterol. Hepatol. Off. Clin. Pract. J. Am. Gastroenterol. Assoc. 2011, 9, 483-489.

21. Rubio, A.; Pigneur, B.; Garnier-Lengline, H.; Talbotec, C.; Schmitz, J.; Canioni, D.; Goulet, O.; Ruemmele, F.M. The efficacy of exclusive nutritional therapy in paediatric Crohn's disease, comparing fractionated oral vs. Continuous enteral feeding. Aliment. Pharmacol. Ther. 2011, 33, 1332-1339.

22. Borrelli, O.; Cordischi, L.; Cirulli, M.; Paganelli, M.; Labalestra, V.; Uccini, S.; Russo, P.M.; Cucchiara, S. Polymeric diet alone versus corticosteroids in the treatment of active pediatric Crohn's disease: A randomized controlled open-label trial. Clin. Gastroenterol. Hepatol. Off. Clin. Pract. J. Am. Gastroenterol. Assoc. 2006, 4, 744-753.

23. Berni Canani, R.; Terrin, G.; Borrelli, O.; Romano, M.T.; Manguso, F.; Coruzzo, A.; D’Armiento, F.; Romeo, E.F.; Cucchiara, S. Short- and long-term therapeutic efficacy of nutritional therapy and corticosteroids in paediatric Crohn's disease. Dig. Liver Dis. 2006, 38, 381-387.

24. Navas-Lopez, V.M.; Blasco-Alonso, J.; Maseri, S.L.; Giron Fernandez-Crehuet, F.; Serrano Nieto, M.J.; Vicioso Recio, M.I.; Sierra Salinas, C. Exclusive enteral nutrition continues to be first line therapy for pediatric Crohn's disease in the era of biologics. Anales de Pediatria 2014, doi:10.1016/j.anpedi.2014.02.027. 
25. Grover, Z.; Muir, R.; Lewindon, P. Exclusive enteral nutrition induces early clinical, mucosal and transmural remission in paediatric Crohn's disease. J. Gastroenterol. 2014, 49, 638-645.

26. Qiu, Y.; Mao, R.; Chen, B.L.; Li, X.H.; He, Y.; Zeng, Z.R.; Li, Z.P.; Chen, M.H. Systematic review with meta-analysis: Magnetic resonance enterography vs. Computed tomography enterography for evaluating disease activity in small bowel Crohn's disease. Aliment. Pharmacol. Ther. 2014, 40, 134-146.

27. Ordas, I.; Rimola, J.; Rodriguez, S.; Paredes, J.M.; Martinez-Perez, M.J.; Blanc, E.; Arevalo, J.A.; Aduna, M.; Andreu, M.; Radosevic, A.; et al. Accuracy of magnetic resonance enterography in assessing response to therapy and mucosal healing in patients with Crohn's disease. Gastroenterology 2014, 146, 374-382.

28. Afzal, N.A.; van Der Zaag-Loonen, H.J.; Arnaud-Battandier, F.; Davies, S.; Murch, S.; Derkx, B.; Heuschkel, R.; Fell, J.M. Improvement in quality of life of children with acute Crohn's disease does not parallel mucosal healing after treatment with exclusive enteral nutrition. Aliment. Pharmacol. Ther. 2004, 20, 167-172.

29. Whitten, K.E.; Leach, S.T.; Bohane, T.D.; Woodhead, H.J.; Day, A.S. Effect of exclusive enteral nutrition on bone turnover in children with Crohn's disease. J. Gastroenterol. 2010, 45, 399-405.

30. Van Loo, E.S.; Dijkstra, G.; Ploeg, R.J.; Nieuwenhuijs, V.B. Prevention of postoperative recurrence of Crohn’s disease. J. Crohns Colitis. 2012, 6, 637-646.

31. Yamamoto, T.; Shiraki, M.; Nakahigashi, M.; Umegae, S.; Matsumoto, K. Enteral nutrition to suppress postoperative Crohn's disease recurrence: A five-year prospective cohort study. Int. J. Colorect. Dis. 2013, 28, 335-340.

32. Navas-Lopez, V.M.; Martin-de-Carpi, J.; Segarra, O.; Garcia-Burriel, J.I.; Diaz-Martin, J.J.; Rodriguez, A.; Medina, E.; Juste, M. Present; prescription of enteral nutrition in pediatric Crohn's disease in spain. Nutric. Hospitalaria 2014, 29, 537-546.

33. Podolsky, D.K. Inflammatory bowel disease. N. Engl. J. Med. 2002, 347, 417-429.

34. Fell, J.M.; Paintin, M.; Arnaud-Battandier, F.; Beattie, R.M.; Hollis, A.; Kitching, P.; Donnet-Hughes, A.; MacDonald, T.T.; Walker-Smith, J.A. Mucosal healing and a fall in mucosal pro-inflammatory cytokine mrna induced by a specific oral polymeric diet in paediatric Crohn's disease. Aliment. Pharmacol. Ther. 2000, 14, 281-289.

35. De Jong, N.S.; Leach, S.T.; Day, A.S. Polymeric formula has direct anti-inflammatory effects on enterocytes in an in vitro model of intestinal inflammation. Dig. Dis. Sci. 2007, 52, 2029-2036.

36. Meister, D.; Bode, J.; Shand, A.; Ghosh, S. Anti-inflammatory effects of enteral diet components on Crohn's disease-affected tissues in vitro. Dig. Liver Dis 2002, 34, 430-438.

37. Edelblum, K.L.; Turner, J.R. The tight junction in inflammatory disease: Communication breakdown. Curr. Opin. Pharmacol. 2009, 9, 715-720.

38. Nahidi, L.; Day, A.S.; Lemberg, D.A.; Leach, S.T. Differential effects of nutritional and non-nutritional therapies on intestinal barrier function in an in vitro model. J. Gastroenterol. 2012, 47, 107-117.

39. Nahidi, L.; Leach, S.T.; Mitchell, H.M.; Kaakoush, N.O.; Lemberg, D.A.; Munday, J.S.; Huinao, K.; Day, A.S. Inflammatory bowel disease therapies and gut function in a colitis mouse model. BioMed. Res. Int. 2013, 2013, doi:10.1155/2013/909613. 
40. Thjodleifsson, B.; Sigthorsson, G.; Cariglia, N.; Reynisdottir, I.; Gudbjartsson, D.F.; Kristjansson, K.; Meddings, J.B.; Gudnason, V.; Wandall, J.H.; Andersen, L.P.; et al. Subclinical intestinal inflammation: An inherited abnormality in Crohn's disease relatives? Gastroenterology 2003, 124, 1728-1737.

41. Hollister, E.B.; Gao, C.; Versalovic, J. Compositional and functional features of the gastrointestinal microbiome and their effects on human health. Gastroenterology 2014, 146, 1449-1458.

42. Morgan, X.C.; Huttenhower, C. Meta'omic analytic techniques for studying the intestinal microbiome. Gastroenterology 2014, 146, 1437-1448.

43. Kuczynski, J.; Lauber, C.L.; Walters, W.A.; Parfrey, L.W.; Clemente, J.C.; Gevers, D.; Knight, R. Experimental and analytical tools for studying the human microbiome. Nat. Rev. Genet. 2012, 13, 47-58.

44. Ursell, L.K.; Haiser, H.J.; van Treuren, W.; Garg, N.; Reddivari, L.; Vanamala, J.; Dorrestein, P.C.; Turnbaugh, P.J.; Knight, R. The intestinal metabolome: An intersection between microbiota and host. Gastroenterology 2014, 146, 1470-1476.

45. Schloss, P.D.; Westcott, S.L.; Ryabin, T.; Hall, J.R.; Hartmann, M.; Hollister, E.B.; Lesniewski, R.A.; Oakley, B.B.; Parks, D.H.; Robinson, C.J.; et al. Introducing mothur: Open-source, platformindependent, community-supported software for describing and comparing microbial communities. Appl. Environ. Microbiol. 2009, 75, 7537-7541.

46. Caporaso, J.G.; Kuczynski, J.; Stombaugh, J.; Bittinger, K.; Bushman, F.D.; Costello, E.K.; Fierer, N.; Pena, A.G.; Goodrich, J.K.; Gordon, J.I.; et al. Qiime allows analysis of high-throughput community sequencing data. Nat. Methods 2010, 7, 335-336.

47. Le Chatelier, E.; Nielsen, T.; Qin, J.; Prifti, E.; Hildebrand, F.; Falony, G.; Almeida, M.; Arumugam, M.; Batto, J.M.; Kennedy, S.; et al. Richness of human gut microbiome correlates with metabolic markers. Nature 2013, 500, 541-546.

48. Kostic, A.D.; Xavier, R.J.; Gevers, D. The microbiome in inflammatory bowel disease: Current status and the future ahead. Gastroenterology 2014, 146, 1489-1499.

49. Human Microbiome Project Consortium. Structure, function and diversity of the healthy human microbiome. Nature 2012, 486, 207-214.

50. Qin, J.; Li, R.; Raes, J.; Arumugam, M.; Burgdorf, K.S.; Manichanh, C.; Nielsen, T.; Pons, N.; Levenez, F.; Yamada, T.; et al. A human gut microbial gene catalogue established by metagenomic sequencing. Nature 2010, 464, 59-65.

51. De Filippo, C.; Cavalieri, D.; Di Paola, M.; Ramazzotti, M.; Poullet, J.B.; Massart, S.; Collini, S.; Pieraccini, G.; Lionetti, P. Impact of diet in shaping gut microbiota revealed by a comparative study in children from europe and rural Africa. Proc. Natl. Acad. Sci. USA 2010, 107, 14691-14696.

52. Ley, R.E.; Hamady, M.; Lozupone, C.; Turnbaugh, P.J.; Ramey, R.R.; Bircher, J.S.; Schlegel, M.L.; Tucker, T.A.; Schrenzel, M.D.; Knight, R.; et al. Evolution of mammals and their gut microbes. Science 2008, 320, 1647-1651.

53. Arumugam, M.; Raes, J.; Pelletier, E.; Le Paslier, D.; Yamada, T.; Mende, D.R.; Fernandes, G.R.; Tap, J.; Bruls, T.; Batto, J.M.; et al. Enterotypes of the human gut microbiome. Nature 2011, 473, 174-180. 
54. Cotillard, A.; Kennedy, S.P.; Kong, L.C.; Prifti, E.; Pons, N.; Le Chatelier, E.; Almeida, M.; Quinquis, B.; Levenez, F.; Galleron, N.; et al. Dietary intervention impact on gut microbial gene richness. Nature 2013, 500, 585-588.

55. Wu, G.D.; Chen, J.; Hoffmann, C.; Bittinger, K.; Chen, Y.Y.; Keilbaugh, S.A.; Bewtra, M.; Knights, D.; Walters, W.A.; Knight, R.; et al. Linking long-term dietary patterns with gut microbial enterotypes. Science 2011, 334, 105-108.

56. David, L.A.; Maurice, C.F.; Carmody, R.N.; Gootenberg, D.B.; Button, J.E.; Wolfe, B.E.; Ling, A.V.; Devlin, A.S.; Varma, Y.; Fischbach, M.A.; et al. Diet rapidly and reproducibly alters the human gut microbiome. Nature 2014, 505, 559-563.

57. Zoetendal, E.G.; von Wright, A.; Vilpponen-Salmela, T.; Ben-Amor, K.; Akkermans, A.D.; de Vos, W.M. Mucosa-associated bacteria in the human gastrointestinal tract are uniformly distributed along the colon and differ from the community recovered from feces. Appl. Environ. Microbiol. 2002, 68, 3401-3407.

58. Kellermayer, R.; Mir, S.A.; Nagy-Szakal, D.; Cox, S.B.; Dowd, S.E.; Kaplan, J.L.; Sun, Y.; Reddy, S.; Bronsky, J.; Winter, H.S. Microbiota separation and C-reactive protein elevation in treatment-naive pediatric granulomatous Crohn disease. J. Pediatr. Gastroenterol. Nutr. 2012, 55, 243-250.

59. Whelan, K.; Judd, P.A.; Preedy, V.R.; Simmering, R.; Jann, A.; Taylor, M.A. Fructooligosaccharides and fiber partially prevent the alterations in fecal microbiota and short-chain fatty acid concentrations caused by standard enteral formula in healthy humans. J. Nutr. 2005, 135, 1896-1902.

60. Miquel, S.; Martin, R.; Rossi, O.; Bermudez-Humaran, L.G.; Chatel, J.M.; Sokol, H.; Thomas, M.; Wells, J.M.; Langella, P. Faecalibacterium prausnitzii and human intestinal health. Curr. Opin. Microbiol. 2013, 16, 255-261.

61. Benus, R.F.; van der Werf, T.S.; Welling, G.W.; Judd, P.A.; Taylor, M.A.; Harmsen, H.J.; Whelan, K. Association between faecalibacterium prausnitzii and dietary fibre in colonic fermentation in healthy human subjects. Br. J. Nutr. 2010, 104, 693-700.

62. Turnbaugh, P.J.; Hamady, M.; Yatsunenko, T.; Cantarel, B.L.; Duncan, A.; Ley, R.E.; Sogin, M.L.; Jones, W.J.; Roe, B.A.; Affourtit, J.P.; et al. A core gut microbiome in obese and lean twins. Nature 2009, 457, 480-484.

63. Karlsson, F.H.; Tremaroli, V.; Nookaew, I.; Bergstrom, G.; Behre, C.J.; Fagerberg, B.; Nielsen, J.; Backhed, F. Gut metagenome in european women with normal, impaired and diabetic glucose control. Nature 2013, 498, 99-103.

64. Qin, J.; Li, Y.; Cai, Z.; Li, S.; Zhu, J.; Zhang, F.; Liang, S.; Zhang, W.; Guan, Y.; Shen, D.; et al. A metagenome-wide association study of gut microbiota in type 2 diabetes. Nature 2012, 490, $55-60$.

65. Hansen, R.; Russell, R.K.; Reiff, C.; Louis, P.; McIntosh, F.; Berry, S.H.; Mukhopadhya, I.; Bisset, W.M.; Barclay, A.R.; Bishop, J.; et al. Microbiota of de-novo pediatric IBD: Increased faecalibacterium prausnitzii and reduced bacterial diversity in Crohn's but not in ulcerative colitis. Am. J. Gastroenterol. 2012, 107, 1913-1922. 
66. Gevers, D.; Kugathasan, S.; Denson, L.A.; Vazquez-Baeza, Y.; Van Treuren, W.; Ren, B.; Schwager, E.; Knights, D.; Song, S.J.; Yassour, M.; et al. The treatment-naive microbiome in new-onset Crohn's disease. Cell. Host Microbe 2014, 15, 382-392.

67. Morgan, X.C.; Tickle, T.L.; Sokol, H.; Gevers, D.; Devaney, K.L.; Ward, D.V.; Reyes, J.A.; Shah, S.A.; LeLeiko, N.; Snapper, S.B.; et al. Dysfunction of the intestinal microbiome in inflammatory bowel disease and treatment. Genome Biol. 2012, 13, doi:10.1186/gb-2012-13-9-r79.

68. Davenport, M.; Poles, J.; Leung, J.M.; Wolff, M.J.; Abidi, W.M.; Ullman, T.; Mayer, L.; Cho, I.; Loke, P. Metabolic alterations to the mucosal microbiota in inflammatory bowel disease. Inflamm. Bowel Dis. 2014, 20, 723-731.

69. Sokol, H.; Pigneur, B.; Watterlot, L.; Lakhdari, O.; Bermudez-Humaran, L.G.; Gratadoux, J.J.; Blugeon, S.; Bridonneau, C.; Furet, J.P.; Corthier, G.; et al. Faecalibacterium prausnitzii is an anti-inflammatory commensal bacterium identified by gut microbiota analysis of crohn disease patients. Proc. Natl. Acad. Sci. USA 2008, 105, 16731-16736.

70. Rajca, S.; Grondin, V.; Louis, E.; Vernier-Massouille, G.; Grimaud, J.C.; Bouhnik, Y.; Laharie, D.; Dupas, J.L.; Pillant, H.; Picon, L.; et al. Alterations in the intestinal microbiome (dysbiosis) as a predictor of relapse after infliximab withdrawal in Crohn's disease. Inflamm. Bowel Dis. 2014, 20, 978-986.

71. Wills, E.S.; Jonkers, D.M.; Savelkoul, P.H.; Masclee, A.A.; Pierik, M.J.; Penders, J. Fecal microbial composition of ulcerative colitis and Crohn's disease patients in remission and subsequent exacerbation. PLoS One 2014, 9, doi:10.1371/journal.pone.0090981.

72. Lionetti, P.; Callegari, M.L.; Ferrari, S.; Cavicchi, M.C.; Pozzi, E.; de Martino, M.; Morelli, L. Enteral nutrition and microflora in pediatric Crohn's disease. JPEN. J. Parenter. Enter. Nutr. 2005, 29 (Suppl. 4), 173-178.

73. Leach, S.T.; Mitchell, H.M.; Eng, W.R.; Zhang, L.; Day, A.S. Sustained modulation of intestinal bacteria by exclusive enteral nutrition used to treat children with Crohn's disease. Aliment. Pharmacol. Ther. 2008, 28, 724-733.

74. Gerasimidis, K.; Bertz, M.; Hanske, L.; Junick, J.; Biskou, O.; Aguilera, M.; Garrick, V.; Russell, R.K.; Blaut, M.; McGrogan, P.; et al. Decline in presumptively protective gut bacterial species and metabolites are paradoxically associated with disease improvement in pediatric Crohn's disease during enteral nutrition. Inflamm. Bowel Dis. 2014, 20, 861-871.

75. Schwiertz, A.; Jacobi, M.; Frick, J.S.; Richter, M.; Rusch, K.; Kohler, H. Microbiota in pediatric inflammatory bowel disease. J. Pediatr. 2010, 157, 240-244.

76. Jia, W.; Whitehead, R.N.; Griffiths, L.; Dawson, C.; Waring, R.H.; Ramsden, D.B.; Hunter, J.O.; Cole, J.A. Is the abundance of Faecalibacterium prausnitzii relevant to Crohn's disease? FEMS Microbiol. Lett. 2010, 310, 138-144.

77. Nagy-Szakal, D.; Mir, S.A.; Ross, M.C.; Tatevian, N.; Petrosino, J.F.; Kellermayer, R. Monotonous diets protect against acute colitis in mice: Epidemiologic and therapeutic implications. J. Pediatr. Gastroenterol. Nutr. 2013, 56, 544-550.

(C) 2014 by the authors; licensee MDPI, Basel, Switzerland. This article is an open access article distributed under the terms and conditions of the Creative Commons Attribution license (http://creativecommons.org/licenses/by/4.0/). 